\title{
A Weighted UTASTAR Method for the Multiple Criteria Decision Making with Interval Numbers
}

\author{
Wen-Tao Xiong \\ School of Mathematics and Statistics, \\ Hubei Engineering University, \\ Xiaogan, China \\ E-mail: xiong_2009@foxmail.com
}

\author{
Jing Cheng \\ School of School of Economics and Management, \\ Hubei Engineering University, \\ Xiaogan, China \\ E-mail: chengjing2003@126.com
}

\begin{abstract}
This paper presents a weighted UTASTAR (WUTASTAR) method to extend the conventional UTASTAR approach for the multiple criteria decision making(MCDM) problem with uncertain interval information. In this method, the criteria weights of all criteria are firstly used to normalize the performance values of all alternatives. Subsequently, two linear programming models are constructed to determine the marginal utility function of each criterion based on the preference preorder in the reference set. Finally, the overall utility interval of each alternative under evaluation with interval information is calculated and the ranking is obtained based on the degree of possiblity between pairs of alternatives. In the end of this paper, an example for evaluating suppliers is used to illustrate the procedure of the proposed WUTASTAR approach.
\end{abstract}

Keywords- UTASTAR method; multiple criteria decision making; interval number

\section{INTRODUCTION}

The UTASTAR method ${ }^{[1]}$ is an improved version of the original UTA(utility additive) approach ${ }^{[2]}$, which can infer one or more piecewise linear utility functions using the linear programming technique. Different from the UTA approach, the UTASTAR method utilized the difference between the marginal utilities of two successive values of each criterion as unknown variable. Besides, it used two errors instead of one error to adjust the overall utility of alternatives according the additive formula.

After the UTASTAR approach was introduced in 1985, several different applications were presented in literature. For instance, Mastorakis and Siskos [3] implemented the UTASTAR method to assess 192 therapeutic categories for investment purposes in the Greek pharmaceutical market. Grigoroudis et al. [4] used the UTASTAR method to aggregate the marginal performance of Key Performance Indicators. Krassadaki et al. [5] applied the UTASTAR method as a means of determining criteria average weights per cluster of students for the multi-criteria clustering approach.

However, some real-life problems may involve uncertain data in the evaluation process. It is hard for the decision makers to estimate their preferences with an exact scale. Many researchers have proposed fuzzy UTASTAR approach to sort the alternatives in the uncertain situations. Patiniotakis et al. [6] presented the UTASTAR method to infer fuzzy utility functions from a partial preorder of options evaluated on multiple criteria, which can handle both crisp and fuzzy evaluation data. Ehsanifar et al. [7] used the UTASTAR model in fuzzy environment for selecting the supplier. Besides, the weight information can be not neglected in some MCDM problems. Considering the weights of all criteria, this paper extends the traditional UTASTAR approach and proposes a WUTASTAR method for the MCDM problem with interval information, where the performance values of alternatives in the reference set are exact, whereas the one of alternatives under evaluation are interval numbers.

The remainder of this paper is organized as follows. Section 2 briefly reviews the arithmetic operations of interval numbers and the concept of degree of possibility. Section 3 proposes a WUTASTAR approach for the MCDM problem with interval uncertain information, where the performance of the alternative under evaluation is expressed by interval numbers. In section 4 , an example is used to illustrate the procedure of proposed WUTASTAR method. Finally, section 5 concludes the paper.

\section{THE BASIC CONCEPT AND OPERATIONS OF INTERVAL NUMBERS}

Definition $1^{[8]} \cdot x$ is a nonnegative interval number, if $x=[\underline{x}, \bar{x}]=\{a \mid 0<\underline{x} \leq a \leq \bar{x}\}$, where $\underline{x}$ and $\bar{x}$ are the crisp real numbers. Especially, $x$ is a nonnegative real number if $\underline{x}=\bar{x}$.

For convenience, all the interval numbers in this paper are nonnegative interval numbers. In order to compare and rank the interval numbers, the arithmetic operations and the degree of possibility are given as below.

$$
\begin{aligned}
& \text { Definition } 2^{[8]} \text {. Let } x=[\underline{x}, \bar{x}], y=[\underline{y}, \bar{y}] \text { be two } \\
& \text { different interval numbers and } k \geq 0 \text {, then } \\
& \qquad x+y=[\underline{x}, \bar{x}]+[\underline{y}, \bar{y}]=[\underline{x}+\underline{y}, \bar{x}+\bar{y}] \\
& x-y=[\underline{x}-\bar{y}, \bar{x}-\underline{y}] \\
& k x=[k \underline{x}, k \bar{x}] \text {. Especially, } k x=0 \text { if } k=0
\end{aligned}
$$


Definition $3^{[8]}$. Let $x=[\underline{x}, \bar{x}]$ and $y=[\underline{y}, \bar{y}]$ be two different interval numbers, then the degree of possibility of $P(x \succeq y)$ is defined as:

$$
P(x \succeq y)=\frac{\max \{0, \bar{x}-\underline{y}\}-\max \{0, \underline{x}-\bar{y}\}}{\bar{x}-\underline{x}+\bar{y}-\underline{y}} .
$$

Similarly, the degree of possibility of $P(y \succeq x)$ can be defined in the same way. That is

$$
\begin{aligned}
P(y \succeq x) & =\frac{\max \{0, \bar{y}-\underline{x}\}-\max \{0, \underline{y}-\bar{x}\}}{\bar{x}-\underline{x}+\bar{y}-\underline{y}} \\
& =1-P(x \succeq y) .
\end{aligned}
$$

\section{THE PROPOSED WEIGHTED UTASTAR APPROACH}

Assume that a MCDM problem consists of $m$ criterion $G=\left\{g_{1}, g_{2}, \ldots, g_{m}\right\}$ and $n$ alternatives $A=\left\{a_{1}, a_{2}, \ldots, a_{n}\right\}$. Let $w_{j}$ be the weight value of the $j$ th criterion $g_{j}$, satisfying $\sum_{j=1}^{m} w_{j}=1$. Without loss of generality, all the criteria are considered as benefit criteria that aim at maximization. Furthermore, suppose that the set of alternatives is divided into two parts: the reference set $A^{E}=\left\{a_{1}, a_{2}, \ldots, a_{t}\right\}$ and the set of alternatives under evaluation $B=A \backslash A^{E}=\left\{a_{t+1}, a_{t+2}, \ldots, a_{n}\right\}$. In the reference set, the alternatives are ranked fully or partially based on the DM's preference. The performance value of each alternative $a_{s}$ under the criterion $g_{j}$ is $x_{s j}$, which is exact. However, the one of $a_{k} \in B$ is uncertain, denoted by the interval number $\left[\underline{x}_{k j}, \bar{x}_{k j}\right], j=1,2, \ldots, m$.

According to the traditional UTASTAR method, a new approach called as WUTASTAR is presented as below. The method utilizes the weight information of all criteria and can deal with the MCDM problem with interval numbers.

Step 1. For each crisp value $x_{s j}$ of the alternative $a_{s} \in A^{E}$ and the interval numbers $\left[\underline{x}_{k j}, \bar{x}_{k j}\right]$ of the alternative $a_{k} \in B$, the normalized values $y_{s j}, \underline{y}_{k j}$ and $\bar{y}_{k j}$ are calculated as:

$$
\begin{gathered}
y_{s j}=\frac{x_{s j}}{\max \left\{x_{s j}, \bar{x}_{k j}\right\}}, \underline{y}_{k j}=\frac{\underline{x}_{k j}}{\max \left\{x_{s j}, \bar{x}_{k j}\right\}}, \bar{y}_{k j}=\frac{\bar{x}_{k j}}{\max \left\{x_{s j}, \bar{x}_{k j}\right\}}, \\
s=1,2, \ldots, t ; k=t+1, t+2, \ldots, n ; j=1,2, \ldots, m .
\end{gathered}
$$

Step 2. Considering the importance of all criteria, the weighted normalized value $z_{s j}$ and interval number $\left[\underline{z}_{k j}, \bar{z}_{k j}\right]$ are calculated as below.

$$
\begin{gathered}
z_{s j}=w_{j} y_{s j}, \underline{z}_{k j}=w_{j} \underline{y}_{k j}, \quad \bar{z}_{k j}=w_{j} \bar{y}_{k j}, \\
s=1,2, \ldots, t ; k=t+1, t+2, \ldots, n ; j=1,2, \ldots, m .
\end{gathered}
$$

where $w_{j}$ is the weight of the criterion $g_{j}$, satisfying $\sum_{j=1}^{m} w_{j}=1$.

Step 3. Let $g_{j}^{*}=\max \left\{\max _{s \in\{1,2, \ldots, t\}}\left\{z_{s j}\right\}, \max _{k \in\{t+1, t+2, \ldots, n\}}\left\{\bar{z}_{k j}\right\}\right\}$ and $g_{j^{*}}=\min \left\{\min _{s \in\{1,2, \ldots, t\}}\left\{z_{s j}\right\}, \min _{k \in\{t+1, t+2, \ldots, n\}}\left\{\underline{z}_{k j}\right\}\right\}$ be the best and the worst level of the criterion $g_{j}$, respectively. And the criterion evaluation scale interval $\left[g_{j^{*}}, g_{j}^{*}\right]$ is divided into $\alpha_{j}-1$ equal intervals $\left[g_{j}^{1}, g_{j}^{2}\right],\left[g_{j}^{2}, g_{j}^{3}\right], \ldots,\left[g_{j}^{\alpha_{j}-1}, g_{j}^{\alpha_{j}}\right]$. Thus, the breakpoints can be calculated using the following formula for each criterion $g_{j}$.

$$
g_{j}^{i}=g_{j^{*}}+\frac{i-1}{\alpha_{j}-1}\left(g_{j}^{*}-g_{j^{*}}\right), i=1,2, \ldots, \alpha_{j} .
$$

Step 4. Using the linear interpolation, the marginal utility of the weighted normalized performance value $z_{s j}$ of alternative $a_{s} \in A$ on the jth criterion can be approximated for $z_{s j} \in\left[g_{j}^{i}, g_{j}^{i+1}\right]$ as:

$$
u_{j}\left(z_{s j}\right)=u_{j}\left(g_{j}^{i}\right)+\frac{z_{s j}-g_{j}^{i}}{g_{j}^{i+1}-g_{j}^{i}}\left(u_{j}\left(g_{j}^{i+1}\right)-u_{j}\left(g_{j}^{i}\right)\right),
$$

where $u_{j}\left(g_{j}^{i}\right)$ is the utility value of breakpoint $g_{j}^{i}$ on the criterion $g_{j}$. Based on the traditional UTASTAR method, let $\xi_{i j}=u_{j}\left(g_{j}^{i+1}\right)-u_{j}\left(g_{j}^{i}\right) \geq 0, i=1,2, \ldots, \alpha_{j}-1$ for each criterion $g_{j}$, which satisfies the following constrains.

$$
\xi_{1 j}=u_{j}\left(g_{j}^{2}\right), j=1,2, \ldots, m, \sum_{j=1}^{m} \sum_{i=1}^{\alpha_{j}-1} \xi_{i j}=1 .
$$

Step 5.Let $\xi_{j}=\left(\xi_{1 j}, \xi_{2 j}, \ldots, \xi_{\alpha_{j}-1, j}\right)^{T}$ and $h_{s j}=(1,1, \ldots, 1$, $\left.\frac{z_{s j}-g_{j}^{i}}{g_{j}^{i+1}-g_{j}^{i}}, 0,0, \ldots, 0\right)^{T}$, for $z_{s j} \in\left[g_{j}^{i}, g_{j}^{i+1}\right], j=1,2, \ldots, m$, where $h_{s j}$ is the $\alpha_{j}-1$ dimension vector and $\frac{z_{s j}-g_{j}^{i}}{g_{j}^{i+1}-g_{j}^{i}}$ is 
in the $i+1$ row. Thus, we can express the global utility of any $a_{s} \in A$ using the following additive formula:

$$
U\left(a_{s}\right)=\sum_{j=1}^{m} u_{j}\left(z_{s j}\right)=\sum_{j=1}^{m} h_{s j}^{T} \cdot \xi_{j}
$$

Generally, there may be the deviation using the additive formula to estimate the utility function of decision maker. Thus, the underestimate error and overestimate error are introduced as below.

$$
U^{\prime}\left(a_{s}\right)=U\left(a_{s}\right)+\sigma_{s}^{-}-\sigma_{s}^{+} \text {, with } \sigma_{s}^{-}, \sigma_{s}^{+} \geq 0 .
$$

Furthermore, for every pair of the successive alternatives belonging to $a_{s}, a_{s+1} \in A^{E}$, the deviation of them is computed as:

$$
\begin{aligned}
\Delta\left(a_{s}, a_{s+1}\right) & =U\left(a_{s}\right)+\sigma_{s}^{-}-\sigma_{s}^{+}-U\left(a_{s+1}\right)-\sigma_{s+1}^{-}+\sigma_{s+1}^{+} \\
& =U\left(a_{s}\right)-U\left(a_{s+1}\right)+\sigma_{s}^{-}-\sigma_{s+1}^{-}+\sigma_{s+1}^{+}-\sigma_{s}^{+} .
\end{aligned}
$$

Step 6. In multi-attribute utility theory, the utility function $U^{\prime}\left(a_{s}\right)$ is compatible, if $\Delta\left(a_{s}, a_{s+1}\right) \geq \delta$ if $a_{s} \succ a_{s+1}$, and $\Delta\left(a_{s}, a_{s+1}\right)=0$ if $a_{s} \sim a_{s+1}$ for $a_{s}, a_{s+1} \in A^{E}$, where the notation $a_{s} \succ a_{s+1}$ means that $a_{s}$ prefers to $a_{s+1}$ and the notation $a_{s} \sim a_{s+1}$ denotes that $a_{s}$ is indifferent to $a_{s+1} . \delta$ is a positive number to ensure the strict inequality $\Delta\left(a_{s}, a_{s+1}\right)>0$. In order to make the marginal utility function be as consistent as possible with the DM's preference, the sum of all error variables should be minimized. Thus, a linear programming model is constructed as below.

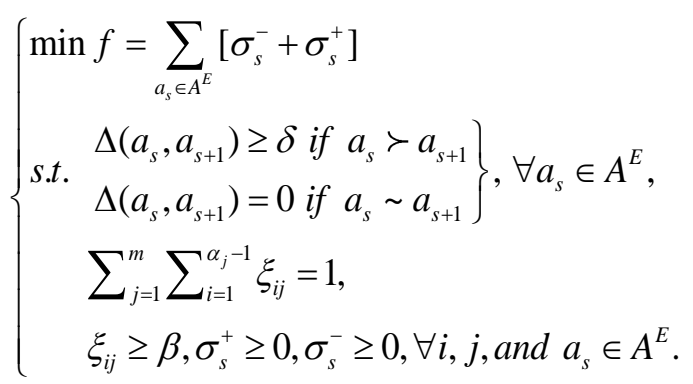

where $\xi_{i j}, \sigma_{s}^{+}, \sigma_{s}^{-}$are the unknown variables, and $\delta$ is an arbitrarily small positive number, $\beta$ can be fixed as 0 .

Step 7. Unfortunately, the optimal solution of the above linear programming is usually non-unique. Thus, the postoptimality analysis is needed to determine the final marginal utility function. Several models have been presented in literature to select an optimal solution. Here, we use the following MP2 model from Beuthe and Scannella [9] to determine the final marginal utility function.

$$
\left\{\begin{array}{l}
\max \delta+\beta \\
\text { s.t. } \sum_{a_{s} \in A^{E}}\left[\sigma_{s}^{-}+\sigma_{s}^{+}\right]=f^{*}, \\
\text { all the constraints in (10). }
\end{array}\right.
$$

where $f^{*}$ is the optimal value of the LP in Step 6 and $\delta$ and $\beta$ are unknown variables .

Step 8. After the marginal utility function is obtained using the linear programming models (10) and (11), we can further calculate the utility interval of each alternative under evaluation. For each alternative $a_{k} \in B$, The utility interval is calculated using the equation (9) based on the point of interval number $\left[\underline{z}_{k j}, \bar{z}_{k j}\right]$.

Step 9. Using the formulas (3) and (4), the degree of possibility of utility intervals obtained in Step 8 is calculated, and then the alternatives under evaluation are ranked according to the degree of possibility. That is, if the degree of possibility of $P\left(a_{s} \succeq a_{s+1}\right) \geq 0.5$, it is considered as the alternative $a_{s}$ prefer the alternative $a_{s+1}$, denoted by

$$
a_{s} \stackrel{P\left(a_{s} \succeq a_{s+1}\right)}{\succeq} a_{s+1} .
$$

\section{AN ILLUSTRATED EXAMPLE}

In this section, the example is adapted from Karande and Chakraborty [10] to illustrate the proposed WUTASTAR method. In their example, five criteria were considered about a supplier selection problem, which were technological capability (TC), conformance quality (CQ), conflict resolution (CR), relationship closeness (RC) and profitability of supplier (PS), respectively. All those criteria were expressed in percentage values and were benefit criteria. The weights of all criteria were $\mathrm{w}_{\mathrm{TC}}=0.3410, \mathrm{w}_{\mathrm{CQ}}=0.2900$, $\mathrm{w}_{\mathrm{CR}}=0.1627, \mathrm{w}_{\mathrm{RC}}=0.1073$ and $\mathrm{w}_{\mathrm{PS}}=0.0990$, respectively. Suppose that all the suppliers are considered as different alternatives. Five suppliers were evaluated in the reference set and their ranking is known, denoted as $a_{1} \succ a_{3} \succ a_{2} \succ a_{5} \succ a_{4}$. Table 1 presents the performance of five alternative or suppliers with respect to all the considered criteria. Different from Karande and Chakraborty [10], five another suppliers is assumed and need further to ranked in this section based on the known ranking in the reference set. Their performance values are denoted as interval numbers and shown in Table II. 
TABLE I. THE PERFORMANCE VALUES OF ALTERNATIVES IN THE REFERENCE SET

\begin{tabular}{c|ccccc}
\hline \multirow{2}{*}{ Weights } & TC & CQ & CR & RC & PS \\
\cline { 2 - 6 } Supplier & 0.3410 & 0.2900 & 0.1627 & 0.1073 & 0.0990 \\
\hline $\mathrm{a}_{1}$ & 55.08 & 87.45 & 65.96 & 67.07 & 50.34 \\
$\mathrm{a}_{2}$ & 60.24 & 76.34 & 47.66 & 53.51 & 33.56 \\
$\mathrm{a}_{3}$ & 67.87 & 66.35 & 51.09 & 60.17 & 44.87 \\
$\mathrm{a}_{4}$ & 47.56 & 58.34 & 66.87 & 43.86 & 29.16 \\
$\mathrm{a}_{5}$ & 53.50 & 60.17 & 38.36 & 40.87 & 56.76 \\
\hline \multirow{2}{*}{ Intervals $\alpha_{j}$} & 4 & 3 & 3 & 2 & 2 \\
\hline
\end{tabular}

TABLE II.THE PERFORMANCE INTERVALS OF THE ALTERNATIVES UNDER EVALUATION

\begin{tabular}{c|ccccc}
\hline Supplier & TC & CQ & CR & RC & PS \\
\hline $\mathrm{a}_{6}$ & {$[53.37,56.71]$} & {$[60.55,82.38]$} & {$[41.40,42.06]$} & {$[50.08,56.65]$} & {$[33.64,38.82]$} \\
$\mathrm{a}_{7}$ & {$[49.26,62.93]$} & {$[59.91,74.01]$} & {$[54.58,65.78]$} & {$[47.76,62.64]$} & {$[34.59,51.08]$} \\
$\mathrm{a}_{8}$ & {$[52.21,62.87]$} & {$[73.79,87.33]$} & {$[38.49,51.75]$} & {$[56.20,58.01]$} & {$[36.09,37.75]$} \\
$\mathrm{a}_{9}$ & {$[55.29,66.11]$} & {$[60.62,81.02]$} & {$[38.69,60.45]$} & {$[55.27,58.93]$} & {$[43.75,46.16]$} \\
$\mathrm{a}_{10}$ & {$[50.65,59.09]$} & {$[71.23,85.53]$} & {$[47.97,61.66]$} & {$[60.47,64.90]$} & {$[33.73,42.22]$} \\
\hline
\end{tabular}

TABLE III. WEIGHTED NORMALIZED OF ALL ALTERNATIVES UNDER THE CONSIDERED CRITERIA

\begin{tabular}{|c|c|c|c|c|c|}
\hline Supplier & $\mathrm{TC}$ & CQ & $\mathrm{CR}$ & $\mathrm{RC}$ & PS \\
\hline $\mathrm{a}_{1}$ & 0.2767 & 0.2900 & 0.1605 & 0.1073 & 0.0878 \\
\hline $\mathrm{a}_{2}$ & 0.3027 & 0.2532 & 0.1160 & 0.0856 & 0.0585 \\
\hline$a_{3}$ & 0.3410 & 0.2200 & 0.1243 & 0.0963 & 0.0783 \\
\hline $\mathrm{a}_{4}$ & 0.2390 & 0.1935 & 0.1627 & 0.0702 & 0.0509 \\
\hline$a_{5}$ & 0.2688 & 0.1995 & 0.0933 & 0.0654 & 0.0990 \\
\hline$a_{6}$ & {$[0.2681,0.2849]$} & {$[0.2008,0.2732]$} & {$[0.1007,0.1023]$} & {$[0.0801,0.0906]$} & {$[0.0587,0.0677]$} \\
\hline$a_{7}$ & {$[0.2475,0.3162]$} & {$[0.1987,0.2454]$} & {$[0.1328,0.1600]$} & {$[0.0764,0.1002]$} & {$[0.0603,0.0891]$} \\
\hline $\mathrm{a}_{8}$ & {$[0.2623,0.3159]$} & {$[0.2447,0.2896]$} & {$[0.0936,0.1259]$} & {$[0.0899,0.0928]$} & {$[0.0629,0.0658]$} \\
\hline $\mathrm{a}_{9}$ & {$[0.2778,0.3322]$} & {$[0.2010,0.2687]$} & {$[0.0941,0.1471]$} & {$[0.0884,0.0943]$} & {$[0.0763,0.0805]$} \\
\hline$a_{10}$ & {$[0.2545,0.2969]$} & {$[0.2362,0.2836]$} & {$[0.1167,0.1500]$} & {$[0.0967,0.1038]$} & {$[0.0588,0.0736]$} \\
\hline
\end{tabular}

Utilizing the presented WUTASTAR method, the weighted normalized performance values of all alternatives are shown in Table 3. The most and the least preferred values for each criteria are easily obtained based on Table 3 , which is $0.3410,0.2900,0.1627,0.1073,0.0990$ and $0.2390,0.1935$, $0.0933,0.0654,0.0509$, respectively. The range of each criterion is divided into some equal intervals and the number of intervals is given in the last row in Table 1. Thus, the breakpoints of each criterion are calculated using the formula (7). Subsequently, the following linear programming model is established according to the preference relation in the reference set and the model (10).

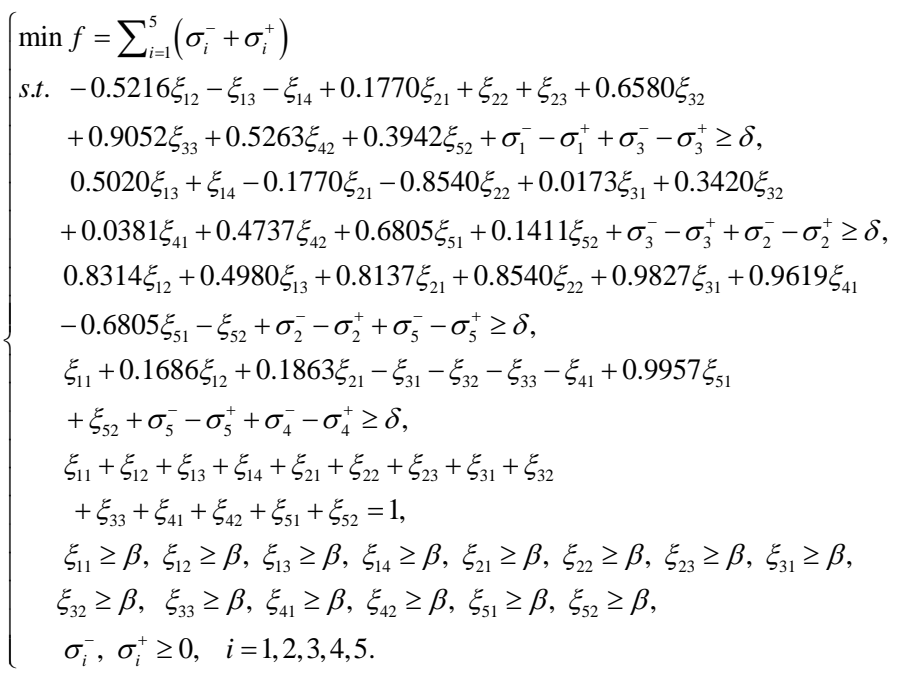


where the value of $\delta$ and $\beta$ are fixed as 0.0001 and 0 , respectively. The linear programming is solved using Lingo software. The optimal objective value is $f^{*}=0$. However, the optimal solution is non-unique. Therefore, the postoptimality analysis is needed. According to the model (11), the optimal results of this linear programming are obtained as: $\varphi^{*}=0.24399, \xi_{11}=0, \xi_{12}=0, \xi_{13}=0, \xi_{14}=0$,

$$
\begin{aligned}
& \xi_{21}=0.3471, \xi_{22}=0, \xi_{23}=0, \xi_{31}=0, \xi_{32}=0, \xi_{33}=0, \\
& \xi_{41}=0.1037, \xi_{42}=0.3462, \xi_{51}=0.2030, \xi_{52}=0 .
\end{aligned}
$$

Using these values, the utility intervals are further calculated for all alternative under evaluation as $\mathrm{U}\left(\mathrm{a}_{6}\right)=[0.2176,0.6633]$, $\mathrm{U}\left(\mathrm{a}_{7}\right)=[0.1904,0.8826], \mathrm{U}\left(\mathrm{a}_{8}\right)=[0.6113,0.6836], \mathrm{U}\left(\mathrm{a}_{9}\right)=[0.4213$, 0.7843], $\mathrm{U}\left(\mathrm{a}_{10}\right)=[0.6898,0.9319]$. Based on the degree of possibility, the final ranking is achieved as:

$$
a_{10} \stackrel{1}{\succ} a_{8} \stackrel{0.6025}{\succ} a_{9} \stackrel{0.5628}{\succ} a_{7} \stackrel{0.5844}{\succ} a_{6} .
$$

\section{CONCLUSION}

The original UTASTAR method dealt with the decision making problem with crisp values. This paper extends the original UTASTAR method from two aspects. On one hand, the weight of each criterion is considered in initial data. All the performance values are weighted normalized, where the cost criterion and benefit criterion can be handled in a unified framework. On the other hand, the alternatives under evaluation are measured with uncertain interval numbers. Utilizing the proposed WUTASTAR method, the utility interval numbers are obtained to rank all the alternatives using the degree of possibility. If the performance value is crisp, the presented WUTASTAR method can be used to compare the alternatives under evaluation straightly.

\section{ACKNOWLEDGMENT}

This work is supported by Scientific Research Program Funded by Hubei Provincial Education Department (No. Q20132706) and National Natural Science Foundation of China (No. 71503073).

\section{REFERENCES}

[1] Y. Siskos, D. Yannacopoulos, "UTASTAR: An ordinal regression method for building additive value functions", Investigacao Operational, vol. 5, no. 1, pp. 39-53, 1985.

[2] E. Jacquet-Lagreze, J. Siskos, "Assessing a set of additive utility functions for multicriteria decision-making, the UTA method", European journal of operational research, vol. 10, no. 2, pp. 151-164, 1982.

[3] K. Mastorakis, E. Siskos, "Value focused pharmaceutical strategy determination with multicriteria decision analysis techniques", Omega. vol. 59, Part A, pp. 84-96, 2015.

[4] E. Grigoroudis, E. Orfanoudaki, C. Zopounidis, "Strategic performance measurement in a healthcare organisation: A multiple criteria approach based on balanced scorecard", Omega, vol. 40, no. 1, pp. 104-119, 2012.

[5] E. Krassadaki, K. Lakiotaki, N.F. Matsatsinis, "Students' behaviour in peer assessment: a multi-criteria clustering approach', European Journal of Engineering Education, vol. 39, no. 3, pp. 233-246, 2014.

[6] I. Patiniotakis, D. Apostolou, G. Mentzas, "Fuzzy UTASTAR: A method for discovering utility functions from fuzzy data", Expert Systems with Applications: An International Journal, vol. 38, no. 12, pp. 15463-15474, 2011

[7] M. Ehsanifar, A.T. Eshlaghi, M.A. Keramati, J. Nazemi, "The Development of UTASTAR Method in Fuzzy Environment for Supplier Selection", European Journal of Scientific Research, vol. 108, no. 3, pp. 317-326, 2013.

[8] Z. Yue, "An extended TOPSIS for determining weights of decision makers with interval numbers", Knowledge-Based Systems, vol. 24, no. 1, pp. 146-153, 2011.

[9] M. Beuthe, G. Scannella, "Comparative analysis of UTA multicriteria methods", European Journal of Operational Research, vol. 130, no. 2, pp. 246-262, 2001.

[10] P. Karande, S. Chakraborty, "Supplier Selection Using Weighted Utility Additive Method", Journal of The Institution of Engineers (India): Series C, vol. 96, pp.1-10, 2015. 